\title{
Comparative analysis of the microRNA transcriptome between yak and cattle provides insight into high-altitude adaptation
}

Jiuqiang Guan $^{1}$, Keren Long ${ }^{2}$, Jideng Ma ${ }^{2}$, Jinwei Zhang ${ }^{2}$, Dafang He ${ }^{2}$, Long Jin ${ }^{2}$, Qianzi Tang ${ }^{2}$, Anan Jiang $^{2}$, Xun Wang ${ }^{2}$, Yaodong Hu ${ }^{2}$, Shilin Tian ${ }^{3}$, Zhi Jiang ${ }^{3}$, Mingzhou Li ${ }^{\text {Corresp.. }}{ }^{2}$, Xiaolin Luo ${ }^{\text {Corresp. } 1}$

${ }^{1}$ Yak Research Institute, Sichuan Academy of Grassland Science, ChengDu, Sichuan, China

2 Institute of Animal Genetics and Breeding, College of Animal Science and Technology, Sichuan Agricultural University, ChengDu, Sichuan, China

3 Novogene Bioinformatics Institute, Beijing, China

Corresponding Authors: Mingzhou Li, Xiaolin Luo

Email address: mingzhou.li@sicau.edu.cn, xiaolinlu08976@163.com

Extensive and in-depth investigations of high-altitude adaptation have been carried out at the level of morphology, anatomy, physiology and genomics, but few investigations focused on the roles of microRNA (miRNA) in high-altitude adaptation. We examined the differences in the miRNA transcriptomes of two representative hypoxia-sensitive tissues (heart and lung) between yak and cattle, two closely related species that live in high and low altitudes, respectively. In this study, we identified a total of 808 mature miRNAs, which corresponded to 715 pre-miRNAs in the two species. The further analysis revealed that both tissues showed relatively high correlation coefficient between yak and cattle, but a greater differentiation was present in lung than heart between the two species. In addition, miRNAs with significantly differentiated patterns of expression in two tissues exhibited cooperation effect in high altitude adaptation based on miRNA family and cluster. Functional analysis revealed that differentially expressed miRNAs were enriched in hypoxia-related pathways, such as the HIF-1 $\alpha$ signaling pathway, the insulin signaling pathway, the PI3KAkt signaling pathway, nucleotide excision repair, cell cycle, apoptosis and fatty acid metabolism, which indicated the important roles of miRNAs in high altitude adaptation. These results suggested the diverse degrees of miRNA transcriptome variation in different tissues between yak and cattle, and suggested extensive roles of miRNAs in high altitude adaptation. 
1 Comparative analysis of the microRNA transcriptome between yak and cattle provides

2

3 Jiuqiang Guan ${ }^{1 \dagger}$, Keren Long ${ }^{2 \dagger}$, Jideng $\mathrm{Ma}^{2 \dagger}$, Jinwei Zhang ${ }^{2}$, Dafang $\mathrm{He}^{2}$, Long Jin ${ }^{2}$, Qianzi

4 Tang $^{2}$, Anan Jiang ${ }^{2}$, Xun Wang 2 , Yaodong $\mathrm{Hu}^{2}$, Shilin Tian, 1,3, Zhi Jiang ${ }^{3}$, Mingzhou Li ${ }^{2 *}$,

5 Xiaolin $\mathrm{Luo}^{1 *}$

$6{ }^{1}$ Yak Research Institute, Sichuan Academy of Grassland Science, Chengdu 611731, China;

$7 \quad 2$ Institute of Animal Genetics \& Breeding, College of Animal Science \& Technology, Sichuan

8 Agricultural University, Wen'jiang 625014, China;

$9 \quad{ }^{3}$ Novogene Bioinformatics Institute, Beijing 100089, China;

Correspondence and requests for materials should be addressed to M.L.

11 (mingzhou.li@sicau.edu.cn) or X.L. (luox12004@sina.com);

$\dagger$ These authors contributed equally to this work. 
25

26

27

\section{Abstract}

Extensive and in-depth investigations of high-altitude adaptation have been carried out at the level of morphology, anatomy, physiology and genomics, but few investigations focused on the roles of microRNA (miRNA) in high-altitude adaptation. We examined the differences in the miRNA transcriptomes of two representative hypoxia-sensitive tissues (heart and lung) between yak and cattle, two closely related species that live in high and low altitudes, respectively. In this study, we identified a total of 808 mature miRNAs, which corresponded to 715 pre-miRNAs in the two species. The further analysis revealed that both tissues showed relatively high correlation coefficient between yak and cattle, but a greater differentiation was present in lung than heart between the two species. In addition, miRNAs with significantly differentiated patterns of expression in two tissues exhibited co-operation effect in high altitude adaptation based on miRNA family and cluster. Functional analysis revealed that differentially expressed miRNAs were enriched in hypoxia-related pathways, such as the HIF-1 $\alpha$ signaling pathway, the insulin signaling pathway, the PI3K-Akt signaling pathway, nucleotide excision repair, cell cycle, apoptosis and fatty acid metabolism, which indicated the important roles of miRNAs in high altitude adaptation. These results suggested the diverse degrees of miRNA transcriptome variation in different tissues between yak and cattle, and suggested extensive roles of miRNAs in high altitude adaptation.

\section{Introduction}

The yak is a unique livestock species that lives in the Qinghai-Tibet Plateau and adjacent areas, at altitudes of 2,500-6,000 m(Nivsarkar et al. 1997). Yak and cattle is a pair of closely related species and diverged five million years ago (Qiu et al. 2012). Although yak and cattle are different species, their genomes have strong similarities, including identical number of chromosomes (30 chromosomes), similar karyotypes (Wiener et al. 2003) and the extensive synteny which cover up to $94 \%$ of the yak genome(Qiu et al. 2012), leading to the availability of these two species in deciphering high altitude adaptation mechanism. Yak have evolved some 
51 anatomical and physiological traits that equip them for the extreme high-altitude environment, 52 including larger lungs and hearts(Wiener et al. 2003), blunted hypoxic pulmonary 53 vasoconstriction(Dolt et al. 2007), strong environmental sense(Wiener et al. 2003) and high 54 energy metabolism(Wang et al. 2011). The mechanism of high altitude adaptation have become

topic of great interests in recent years. Extensive and in-depth investigations of high-altitude adaptation have been carried out at the level of morphology(Hoppeler et al. 1990), anatomy(Ahmad et al. 2016), hemodynamics(Ahmad et al. 2016), physiology(Monge \& LeónVelarde 1991) and genomics(Huertasánchez et al. 2013; Qiu et al. 2012), but few investigations focused on the role of miRNA in high-altitude adaptation.

microRNAs (miRNAs) is a kind of endogenous noncoding RNAs which posttranscriptionally modulates gene expression through either translational repression or mRNA degradation(Bushati \& Cohen 2007). Recent studies identified a large amount of hypoxiainduced miRNAs that widely participate in various biological processes, including cell apoptosis(Lynam-Lennon et al. 2009), DNA repair(Hu \& Gatti 2011; Landau \& Slack 2011), cell cycle(Chen \& $\mathrm{Hu}$ 2012), angiogenesis(Nicoli et al. 2010) and metabolism regulation(Kulshreshtha et al. 2007). For example, myocardial hypoxia induces expression of the miR-199a:214 cluster which thereafter downregulates several cardiac and mitochondrial targets, such as $P P A R \delta$, downregulation of $P P A R \delta$ provokes glycolytic metabolism in heart failure(El et al. 2013).

We investigate: a. whether miRNAs was involved in the mechanism of high altitude adaptation, b. the kind of high altitude-adaptation miRNAs, c. the functions of these high altitude-adaptation miRNAs. We constructed 11 small RNA cDNA libraries of heart and lung tissues in 3 female yaks and 3 female cattle, and performed small RNA sequencing. In total, we identified 757 and 769 unique mature miRNAs in yak and cattle, respectively. The further analysis revealed stronger expression divergence in lung than heart between yak and cattle. In two tissues, we identified total of 85 differentially expressed (DE) miRNAs between yak and cattle, which showed potential co-operation effect in high altitude adaptation. The functional 
enrichment analysis revealed extensive roles of miRNAs in hypoxia-related pathways, such as the HIF-1 $\alpha$ signaling pathway, the insulin signaling pathway, the PI3K-Akt signaling pathway, nucleotide excision repair, cell cycle, apoptosis and fatty acid metabolism.

\section{Materials and methods}

Ethics statement. All animals were conducted according to the Institutional Animal Care and Use Committee in College of Animal Science and Technology, Sichuan Agricultural University, Sichuan, China under permit No. DKY-B20151608. Yak and cattle were collected from A'ba (altitude, $3000 \mathrm{~m}$ ) and Chengdu (altitude, $485 \mathrm{~m}$ ), respectively. Animals were feed with free access to food and water, and were killed humanely.

Sample collection. Three unrelated 2-year old adult females for both of yaks and cattle (Luxi Huang cattle) were used in this study. Two of significant hypoxia-responsive tissues (heart and lung) were rapidly collected from each carcass, washed three times with physiological saline, immediately frozen in liquid nitrogen. All frozen samples were stored at $-80{ }^{\circ} \mathrm{C}$ until RNA extraction.

RNA isolation, library preparation, and sequencing. The total RNA were extracted with Trizol (Ambion, USA). NanoDrop ND-2000 spectrophotometer (Nano Drop, DE, USA) and Bioanalyzer 2100 (Agilent Technologies, Santa Clara, CA, USA) were used to monitor the concentration and integrity of RNA, respectively. In brief, several successive steps consist the Illumina sequencing. The small RNA with length of 14-40 nt were first purified by polyacrylamide gel electrophoresis (PAGE), and then specific adapters were ligated to the purified small RNA. The ligated RNA were reverse transcribed to cDNA libraries. Finally, each library were sequenced on Genome Analyzer.

Data analysis of small RNA sequencing. The analysis procedure of miRNA identification and quantification was conducted as previous study ( $\mathrm{Li}$ et al. 2010) with several modifications. The raw reads were processed using Illumina's Genome Analyzer Pipeline software. After trimming 
103

104

105

106

107

108

109

110

111

112

113

114

115

116

off the adapters, the resulting reads was successively filtered by some strict criterial ( only reads with size ranging from 14 to $27 \mathrm{nt}$, containing no more than $80 \% \mathrm{~A}, \mathrm{C}, \mathrm{G}$ or $\mathrm{T}$; containing no more than two $\mathrm{N}$ (undetermined bases) were retained). Bovine known classes of RNAs (i.e. mRNA, rRNA, tRNA, snRNA, snoRNA and repeats) were subsequently removed through searching against three databases, including the NCBI(Pruitt et al. 2009), Rfam(Gardner et al. 2008) and Repbase(Lukasz et al. 2006). All retained reads were defined as "high quality reads". And then, "high quality reads" were mapped to bovine genome and mapped reads were retained. All retained "high quality reads" were mapped to the known bovine pre-miRNAs and known pre-miRNAs from 24 other mammals in miRBase 20.0(Kozomara \& Griffithsjones 2014), reads mapping to bovine or other mammalian miRNAs were defined as miRNA candidates. Given the reliability of miRNA identification, miRNA candidates with a read count $\geq 3$ at least one sample were retained for subsequently analysis. Comparable numbers of miRNA species and similar expressional patterns were identified for using bovine genome or yak genome (Figure S1), we selected bovine genome as the reference genome in this study.

Identification of differentially expressed miRNAs. To reveal the difference of miRNA transcriptome between yak and cattle, we identified the differentially expressed (DE) miRNAs using edgeR package(Robinson et al. 2010). To obtain the normalized expression levels, read counts were loaded into edgeR and normalized using the supplied trimmed-mean-of-M-values algorithm. DE miRNAs are defined as those miRNAs with a folder change $>2$ and a Benjamini Hochberg FDR (False Discovery Rate) $<0.05$ between yak and cattle.

The Prediction and functional analysis of miRNA target genes. As a miRNA pathway analysis web-server, DIANA-mirPath(Vlachos et al. 2015) is able to identify miRNA target, and molecular Gene Ontology (GO) and pathways (http://www.microrna.gr/miRPathv3). The predictions in this study were based on the database of human beings, owing to the lack of the bovine miRNAs in mirPath3.0.

\section{Results}


129

130

131

132

133

134

135

136

137

138

139

140

141

142

143

144

145

146

147

148

149

150

151

152

153

154

\section{Overview of small-RNA sequencing}

We constructed and sequenced 11 small RNA libraries, including five heart libraries (i.e. two yak hearts and three cattle hearts) and six lung libraries (i.e. three yak lungs and three cattle lungs). A total of 96.19 million (M) raw reads were obtained from the 11 libraries, with an average of $8.74 \mathrm{M}(8.74 \pm 3.37 \mathrm{M})$ in each library. Through a series of strict criteria, a total of 84.57 $\mathrm{M}$ raw reads were defined as high quality reads; With an average of $7.69 \pm 2.68 \mathrm{M}$ raw reads in each library were defined as "high-quality reads" (Table S1). By aligning these data to the Rfam database, we also identified abundant known non-coding RNAs (e.g. tRNAs, rRNAs, snoRNAs and snRNAs) (Fig. S1). Consistent with the canonical size of Dicer-processed products, over $80 \%$ of high-quality reads were $21-23 \mathrm{nt}$ in length $(81.03 \pm 1.72 \%, \mathrm{n}=11)$, and the highest percentage of high-quality reads were $23 \mathrm{nt}$ in length $(45.17 \pm 1.62 \%, \mathrm{n}=11)$, followed by $23 \mathrm{nt}(19.09 \pm 2.25 \%, \mathrm{n}=11)$ and $21 \mathrm{nt}(16.68 \pm 1.65 \%, \mathrm{n}=11)$ (Fig S1). We then examined small RNA profiling of heart and lung for cattle and yak. In total, we identified 808 mature miRNAs, corresponding to 715 pre-miRNAs (Fig. 1A). Majority of these mature miRNAs were widely expressed in all sample and only a few were expressed as tissue or speciesspecific (Fig. 1B). The biological replicates showed high Spearman's correlation coefficient (average $r=0.99$ ), which indicates strong experimental confidence (Fig. S3).

\section{Different organs showed varying degrees of miRNA expression divergence}

To obtain an overview of miRNA transcription patterns, we performed a clustering analysis of the expression of 718 co-expressed miRNAs. As expected, all samples were firstly clustered by tissue and thereafter by species (Fig. 2A), which indicates the longer evolutional history of these organs than the species. In addition, we also conducted principal component analysis. The first eigenvector distinguished tissue differentiation, while the second eigenvector captured the biological differentiation and high altitude-related differentiation between yak and cattle for heart and lung tissues. Lung showed larger differentiation than heart between yak and cattle (Fig. 2B). This result is consistent with the universal organ-specific pattern of mRNA gene 
155

156

157

158

159

160

161

162

163

164

165

166

167

168

169

170

171

172

173

174

175

176

177

178

179

180

181

expression(Brawand \& Al 2011; Melé et al. 2015). It was previously reported that heart showed stronger mRNA transcriptome variance than lung between yak and cattle(Wang et al. 2015), but the miRNA transcriptome showed the opposite trend in this study, which indicates miRNA and mRNA transcriptome inconsistence. Although organ divergence contributes to the primary variance, heart and lung showed similar expression profiles of the most abundant miRNAs (Fig. 2C). Overall, the two organs shares five miRNAs of the top 10 expressed miRNA, which included bta-miR-143-3p, bta-miR-22-3p, bta-miR-27b-3p, bta-miR-30e-5p and bta-miR-30a-5p.

\section{Differentially expressed miRNAs showed a potential co-operation effect in high-altitude} adaptation

To further reveal the miRNA adaptation of yak to high altitudes, we performed differential analysis for lung and yak between yak and cattle. In total, we detected 85 differentially expressed mature miRNAs between yak and cattle (fold change $>2$, FDR $<0.05$ ), of which 29 were present in heart (Fig. 3A) and 70 in lung (Fig. 3B). The detailed information is listed in Table 1 and Table S2, S3. The greater amount of DE lung miRNAs is consistent with the lower Spearman's correlation coefficient for lung, increases the reliability of greater miRNA transcriptome divergence of lung than heart between yak and cattle.

It is well known that miRNAs that derive from a common ancestor can be grouped into a family, and family members often have similar biological function. Of these 85 differentially expressed miRNAs, we detected 6 miRNA families, including miR-2285, miR-34, miR-192, miR-449, miR-200 families (Table S4). We also detected 8 miRNA clusters in differentially expressed miRNAs that included from two members to 13 members, such as the mir-215/206, mir-127/136 cluster (Table S5). The largest cluster was mir-154/376/379 cluster, which consisted of 13 members. To explore the expression pattern of miRNA families and clusters, we calculated the Pearson's correlation coefficient of each family, cluster and background. The background showed a wide Pearson's correlation range from -0.99 to 0.99 , with an average of $0.1221(r=0.1221 \pm 0.55, n=257,403)$. Compared with background, the miRNA families and clusters showed high levels of Pearson's correlation, with coefficients of 0.86 in miRNA clusters 
$182(r=0.86 \pm 0.23, n=478)$ and $0.44(r=0.45 \pm 0.53, n=52)$ in miRNA families (Fig. 3C), 183 which indicated the co-expression and potential similar functions of members in each miRNA 184 family or cluster. These results are consistent with the observation that genes which cluster together share common functions and similar expression pattern(Eisen et al. 1998). Consistent with the hypoxic high-altitude environment, these miRNA families and clusters showed high relevance to high altitude adaptation. For example, the p53-responsive microRNA miR-192 and miR-215 are able to induce cell cycle arrest (Braun et al. 2008) and significantly inhibited cellular nucleotide excision repair (NER)(Xie et al. 2011). We identified two differentially expressed miRNAs not only belonging to the same family but also belonging to the same cluster. These miRNAs included miR-34b/34c/449a/449b, miR-200a/429. The miR-34 family consist of four members, including miR-34a, miR-34b, miR-449a, miR-449b. miR-34a/b family clustered together, and miR-449a/b family clustered together (Fig. 3D,E), and showed similar expression pattern between yak and cattle. miRNAs in each family showed overall similar expression pattern in heart and lung tissues between yak and cattle, indicating the potential similar effects of these miRNAs in high-altitude adaptation. The miR-34 family members showed hypoxia related functions, overexpression of miR-34a is able to regulate the G1/S checkpoint through targeting several genes, such as CDK6 and E2F, et al. (Chen \& Hu 2012; Sun et al. 2008), and miR-34c affects the S-phase checkpoint through binding to c-myc(Cannell \& Bushell 2010). In brief, the similar expression pattern of miRNA families and clusters suggested the potential collaboration effect of miRNAs in high altitude adaptation.

\section{DE miRNAs are associated with hypoxia-related functions}

To illustrate the potential function of identified DE miRNAs, we therefore performed target prediction and functional enrichment analysis by DIANA online software(Vlachos et al. 2015). As expected, the majority of DE miRNAs were involved in hypoxia-related pathways, such as the HIF-1 $\alpha$ signaling pathway, the insulin signaling pathway, the PI3K-Akt signaling pathway, nucleotide excision repair, cell cycle, apoptosis, fatty acid metabolism, which were high relevant to high-altitude adaptation (Fig. 4A, 4B). In particular, the insulin signaling pathway, fatty acid 
209 biosynthesis, the p53 signaling pathway and cell cycle were simultaneously enriched in both 210 tissues, which indicates the central role of these DE miRNAs in high-altitude adaptation 211 pathways.

212 As a sequence-specific transcription factor, p53 regulates the expression of several genes 213 associated with growth arrest (Kracikova et al. 2013), DNA repair, apoptosis, and 214 angiogenesis(Farhang et al. 2013). In this study, we identified 31 DE miRNAs that enriched in 215 P53 signaling pathway, of which 14 was presented in heart and 22 in lung (Table 2). Especially, 216 five miRNAs showed differential expression in both heart and lung, including miR-192-5p, miR217 32-3p, miR-200b-3p, miR-146b-5p, miR-122-5p. We interestingly found that miR-192, miR-32, 218 miR-122, miR-200b are regulators of p53 feedback circuit (Figure 4C). For example, miR-32 is 219 in a recurrent autoregulatory circuit in which p53 regulate the expression of miR-32, and on 220 other hand, miR-32 indirectly increase p53 accumulation through targeting Mdm2 and TSC1 221 (Suh et al. 2012). In addition, p53 can posttranscriptionally regulated the expression of miR-122, 222 and in turn miR-122 can suppress p53 activity by activating Akt(Manfè et al. 2012), thus 223 constructing a negative feedback. Three (miR-192-5p, miR-32-3p, miR-122-5p) of these five 224 miRNAs showed decreased expression level in yak, and one miRNA (miR-125-5p) was 225 upregulated in yak. The differential expression of these p53 feedback circuit regulators may play 226 an important roles in the p53 accumulation.

227 It is well known that some intrinsic and extrinsic genotoxic stress such as oxidative stress and ultraviolet light could result in DNA damage(Jackson \& Bartek 2009). Because of the hypoxia and intensive ultraviolet environment at high altitudes, yak could have evolved strong 230 abilities to resist DNA damage caused by hypoxia and intensive ultraviolet. By conducting a 231 literature search, we identified 10 known DNA damage response miRNAs, including miR-504232 5p, miR-34a, miR-215, miR-192, miR-128, miR-181, et al (Fig. S4A). These miRNAs are 233 involved in many facets of DNA damage response, including DNA repair, the cell cycle 234 checkpoint and apoptosis. It was reported that miR-181a and miR-181b were upregulated in 235 aggressive breast cancers, and their expression level inversely correlates with ataxia 
236

237

238

239

240

241

242

243

244

245

246

247

248

249

250

251

252

253

254

255

256

257

258

259

260

261

262

telangiectasia mutated (ATM) levels and DNA damage repair(Bisso et al. 2013). As a negative regulator of human p53, miR-504 is able to directly bind to two target sites in the p53 3'untranslated region, and play important roles in the process of p53-mediated cell-cycle arrest and apoptosis(Hu et al. 2010). The functional enrichment analysis by DIANA software indicated that target genes of 28 DE miRNAs in lung were also enriched in nucleotide excision repair, which indicates a substantial involvement of DE miRNAs in DNA damage response (Fig. S4B).

HIF-1 $\alpha$ is a basic transcription factor that trans-activates genes which encode proteins that participate in homeostatic responses to hypoxia, it include expression of proteins involving in glucose metabolism(Remels et al. 2015), cell proliferation(Scaringi et al. 2013), and vascularization(Nicolas Skuli 2012; Zhao et al. 2012). In this study, we detected 15 HIF-1 $\alpha$ signaling related miRNAs in heart, and these miRNAs can regulate both upstream and downstream genes of the HIF-1 $\alpha$ signaling pathway (Fig. 4D). It was reported that miR-429 construct a negative regulatory loop with HIF-1 and decrease HIF- 1 activity through targeting HIF1A mRNA before HIF-2 is activated (Bartoszewska et al. 2015). MiR-326, a recognized tumor-suppressing miRNA, has been found to target the 3'-UTR of the human CCND1 mRNA and promote cell apoptosis(Sun et al. 2016). Hypoxia-induced microRNA-424 promoted angiogenesis both in vitro and in vivo, and increased the expression levels of HIF-1 $\alpha$ and HIF-2 $\alpha$ by targeting cullin 2 (CUL2), a key protein for the assembly of the ubiquitin ligase system(Ghosh et al. 2010). In addition, miR-218 repression increases the abundance and activity of multiple RTK effectors, and promotes the activation of hypoxia-inducible factor (HIF), most notably HIF2 $\alpha$ (Mathew et al. 2014).

Some metabolism-related DE miRNAs and pathways were also revealed in this study. For example, the DNA damage causing miR-181a inhibit the expression of genes that were associated with lipid synthesis and increase the expression of genes that were associated with $\beta$ oxidation. It is able to cause decreased expression of isocitrate dehydrogenase 1 which plays roles in tricarboxylic acid (TCA) cycle, thereafter inhibits lipid accumulation(Chu et al. 2015). These results are consistent with the energy shift from fatty acid oxidation to glucose oxidation 
263

264

265

266

267

268

269

270

271

272

273

274

275

276

277

278

279

280

281

282

283

284

285

286

287

288

289

and glycolysis in the high-altitude living conditions(Ding et al. 2014; Ge et al. 2012).

\section{Discussion}

In recent years, the mechanism of high altitude adaptation have become topic of great interests and attracted many specialists from various areas. They carried out extensive and indepth investigations of high altitude adaptation at the levels of morphology(Hoppeler et al. 1990), physiology(Monge \& León-Velarde 1991) and genomics(Huertasánchez et al. 2013; Qiu et al. 2012) in human and other species. Yak and cattle is a pair of closely related species and diverged five million years ago(Qiu et al. 2012), and provide an ideal model for deciphering the mechanism of high altitude adaptation for their high similarity of genome. Here, we performed transcriptome analysis in yak and cattle for two hypoxia-sensitive tissues, heart and lung. We identified comparable numbers of miRNA species using yak and cattle genome, respectively. In addition, we also detected similar expressional patterns across all libraries using yak and cattle genome, with the same heat map matrix of pairwise Spearman's correlations.

In this work, we identified 808 mature miRNAs, corresponding to 715 pre-miRNAs, covering majority of bovine miRNA in miRBase 20.0. The Spearman's correlation analysis revealed that lung showed larger differentiation than heart between yak and cattle at the level of miRNA transcriptome, which is inconsistent with previous study that heart showed stronger variance than lung between yak and cattle at mRNA transcriptome(Wang et al. 2015). We identified a large amount of differentially expressed miRNA between yak and cattle, including 29 miRNAs in heart and 70 miRNAs in lung. Further examination revealed that majority of differentially expressed miRNAs are downregulated in yak, including 18 of 29 DE miRNAs in heart and 48 of 70 DE miRNAs in lung (Table 1). The pervasive downregulation of differentially expressed miRNAs in yak may correspond to the upregulation of target pathways. For example, we detected 15 differentially expressed miRNAs enriched in HIF1 $\alpha$ signaling pathway in heart (Figure 4D), and 13 miRNAs were downregulated in yak which may result in the overall upregulation of HIF1 $\alpha$ signaling pathway, consistent with previous studies that HIF pathway genes played important roles in high altitude adaptation and underwent positive 
290

291

292

293

294

295

296

297

298

299

300

301

302

303

304

305

306

307

308

309

310

311

312

313

314

315

selection in high altitude populations in humans(Beall et al. 2010; Simonson et al. 2010) and other species(Li et al. 2013; Qiu et al. 2012). In addition, we also identified 14 miRNAs were differentially expressed genes in heart and enriched in p53 signaling pathway, of which 13 miRNAs are downregulated in heart. It was reported that alleles of ubiquitin specific peptidase 7 (USP7), interleukin 6 family cytokine (LIF), and murine double minute2 (MDM2) were selected for in relation to harsh environmental variables related to high altitude(Jacovas et al. 2015). The widespread of differentially expressed miRNAs targeting p53 pathway gene indicated the potential involvement of p53 pathway in high altitude adaptation. Taken together, these results indicated the potential roles of these DE miRNAs in yak high altitude adaptation through upregulating important pathways.

Although we found substantial differentially expressed miRNA that may be associated with high altitude adaptation, some limitations in this study should be concerned. Since diverging five million years ago, yak and cattle underwent distinct selective pressure resulting in inherent genetic difference which may to some extent disturbance the result and explanation. In addition, human miRNA orthologs were used to perform miRNA target prediction which may suffer from the miRNA-mRNA interaction bias in different species. A more comprehensive association study of mRNA and miRNA expression is necessary for better revealing the authentic roles of differentially expressed miRNAs.

\section{Conclusions}

In this study, we illustrated the differences in the miRNA transcriptomes for heart and lung between yak and cattle, and suggested extensive roles of miRNAs in high altitude adaptation. The work performed here will provide a typical demonstration for future deciphering the mechanism of high altitude adaptation.

\section{Acknowledgements}

We thank YuJie Wang, Wanling Qiu, Zihu Hu and Can Liu for help with experiments.

\section{Additional information and declaration}


316 Supplementary information accompanied this paper can see supplementary Figure S1-S4 and

317

318

319

320

321

322

323

324

325

326

327

328

329

330

331

332

333

334

335

336

337

338

339

340

341

342

343

344

345

346

347

348

349

350

351

352

353

Table S1-S4.

Accessions codes: The small RNA sequencing reads have been deposited into the NCBI gene expression omnibus (GEO) under the accession number GSE87833.

\section{Reference}

Ahmad KS, Hameed M, Fatima S, Ashraf M, Ahmad F, Naseer M, and Akhtar N. 2016. Morpho-anatomical and physiological adaptations to high altitude in some Aveneae grasses from Neelum Valley, Western Himalayan Kashmir. Acta Physiologiae Plantarum 38:1-14.

Bartoszewska S, Kochan K, Piotrowski A, Kamysz W, Ochocka RJ, Collawn JF, and Bartoszewski R. 2015. The hypoxia-inducible miR-429 regulates hypoxia-inducible factor- $1 \alpha$ expression in human endothelial cells through a negative feedback loop. Faseb Journal 29:1467-1479.

Bisso A, Faleschini M, Zampa F, Capaci V, Santa JD, Santarpia L, Piazza S, Cappelletti V, Daidone M, and Agami R. 2013. Oncogenic miR-181a/b affect the DNA damage response in aggressive breast cancer. Cell cycle (Georgetown, Tex) 12:1679-1687.

Braun CJ, Zhang X, Savelyeva I, Wolff S, Moll UM, Schepeler T, Ørntoft TF, Andersen CL, and Dobbelstein M. 2008. p53-Responsive MicroRNAs 192 and 215 Are Capable of Inducing Cell Cycle Arrest. Cancer Research 68:10094-10104.

Brawand D, and Al E. 2011. The evolution of gene expression levels in mammalian organs. Nature 478:343.

Bushati N, and Cohen SM. 2007. microRNA functions. Annual Review of Cell \& Developmental Biology 23:175-205.

Cannell IG, and Bushell M. 2010. Regulation of Myc by miR-34c: A mechanism to prevent genomic instability? Cell Cycle 9:2726.

Chen F, and $\mathrm{Hu}$ SJ. 2012. Effect of microRNA-34a in cell cycle, differentiation, and apoptosis: A review. Journal of Biochemical \& Molecular Toxicology 26:79-86.

Chu B, Wu T, Lin M, Mei Y, and Wu M. 2015. MiR-181a regulates lipid metabolism via IDH1. Scientific Reports 5:8801.

Ding XZ, Liang CN, Guo X, Wu XY, Wang HB, Johnson KA, and Yan P. 2014. Physiological insight into the high-altitude adaptations in domesticated yaks ( Bos grunniens ) along the Qinghai-Tibetan Plateau altitudinal gradient. Livestock Science 162:233-239.

Dolt KS, Mishra MK, Karar J, Baig MA, Ahmed Z, and Pasha MA. 2007. cDNA cloning, gene organization and variant specific expression of HIF-1 alpha in high altitude yak (Bos grunniens). Gene 386:73-80.

Eisen MB, Spellman PT, Brown PO, and Botstein D. 1998. Cluster analysis and display of genome-wide expression patterns. Proceedings of the National Academy of Sciences of the United States of America 95:14863-14868. 
354

355

356

357

358

359

360

361

362

363

364

365

366

367

368

369

370

371

372

373

374

375

376

377

378

379

380

381

382

383

384

385

386

387

388

389

390

391

392

393

394

El AH, Leptidis S, Dirkx E, Hoeks J, Van BB, Brand K, Mcclellan EA, Poels E, Sluimer JC, and $\mathrm{Mm}$ VDH. 2013. The hypoxia-inducible microRNA cluster miR-199a 214 targets myocardial PPAR $\delta$ and impairs mitochondrial fatty acid oxidation. Cell Metabolism 18:341-354.

Farhang GM, Goossens S, Nittner D, Bisteau X, Bartunkova S, Zwolinska A, Hulpiau P, Haigh K, Haenebalcke L, and Drogat B. 2013. p53 promotes VEGF expression and angiogenesis in the absence of an intact p21-Rb pathway. Cell Death \& Differentiation 20:888-897.

Gardner PP, Daub J, Tate JG, Nawrocki EP, Kolbe DL, Lindgreen S, Wilkinson AC, Finn RD, Griffithsjones S, and Eddy SR. 2008. Rfam: updates to the RNA families database. Nucleic Acids Research 37:D136-140.

Ge RL, Simonson TS, Cooksey RC, Tanna U, Qin G, Huff CD, Witherspoon DJ, Xing J, Bai Z, and Prchal JT. 2012. Metabolic insight into mechanisms of high-altitude adaptation in Tibetans. Molecular Genetics \& Metabolism 106:244-247.

Ghosh G, Subramanian IV, Adhikari N, Zhang X, Joshi HP, Basi D, Chandrashekhar YS, Hall JL, Roy S, and Zeng Y. 2010. Hypoxia-induced microRNA-424 expression in human endothelial cells regulates HIF- $\alpha$ isoforms and promotes angiogenesis. Journal of Clinical Investigation 120:4141-4154.

Hoppeler H, Kleinert E, Schlegel C, Claassen H, Howald H, Kayar SR, and Cerretelli P. 1990. Morphological adaptations of human skeletal muscle to chronic hypoxia. International Journal of Sports Medicine 11 Suppl 1:S3-9.

$\mathrm{Hu}$ H, and Gatti RA. 2011. MicroRNAs: new players in the DNA damage response. Journal of Molecular Cell Biology 3:151-158.

$\mathrm{Hu}$ W, Chan CS, Wu R, Zhang C, Sun Y, Song JS, Tang LH, Levine AJ, and Feng Z. 2010. Negative Regulation of Tumor Suppressor p53 by MicroRNA miR-504. Molecular Cell 38:689-699.

Huertasánchez E, Degiorgio M, Pagani L, Tarekegn A, Ekong R, Antao T, Cardona A, Montgomery HE, Cavalleri GL, and Robbins PA. 2013. Genetic Signatures Reveal HighAltitude Adaptation in a Set of Ethiopian Populations. Molecular Biology \& Evolution 30:1877-1888.

Jackson SP, and Bartek J. 2009. The DNA-damage response in human biology and disease. Nature 461:1071-1078.

Kozomara A, and Griffithsjones S. 2014. miRBase: annotating high confidence microRNAs using deep sequencing data. Nucleic Acids Research 42:68-73.

Kracikova M, Akiri G, George A, Sachidanandam R, and Aaronson SA. 2013. A threshold mechanism mediates p53 cell fate decision between growth arrest and apoptosis. Cell Death \& Differentiation 20:576-588.

Kulshreshtha R, Ferracin M, Negrini M, Calin GA, Davuluri RV, and Ivan M. 2007. Regulation of microRNA Expression: the Hypoxic Component. Cell Cycle 6:1426-1431.

Landau DA, and Slack FJ. 2011. MicroRNAs in Mutagenesis, Genomic Instability, and DNA Repair. Seminars in Oncology 38:743-751. 
395

396

397

398

399

400

401

402

403

404

405

406

407

408

409

410

411

412

413

414

415

416

417

418

419

420

421

422

423

424

425

426

427

428

429

430

431

432

433

434

435

Li M, Xia Y, Gu Y, Zhang K, Lang Q, Chen L, Guan J, Luo Z, Chen H, and Li Y. 2010. MicroRNAome of Porcine Pre- and Postnatal Development. PLoS One 5:e11541.

Lukasz H, Gentles AJ, Oleksiy K, and Jerzy J. 2006. Annotation, submission and screening of repetitive elements in Repbase: RepbaseSubmitter and Censor. BMC Bioinformatics $7: 474$.

Lynam-Lennon N, Maher SG, and Reynolds JV. 2009. The roles of microRNA in cancer and apoptosis. Biological Reviews 84:55.

Manfè V, Biskup E, Rosbjerg A, Kamstrup M, Skov AG, Lerche CM, Lauenborg BT, Ødum N, and Gniadecki R. 2012. miR-122 Regulates p53/Akt Signalling and the ChemotherapyInduced Apoptosis in Cutaneous T-Cell Lymphoma. Plos One 7:e29541.

Mathew LK, Skuli N, Mucaj V, Lee SS, Zinn PO, Sathyan P, Imtiyaz HZ, Zhang Z, Davuluri RV, and Rao S. 2014. miR-218 opposes a critical RTK-HIF pathway in mesenchymal glioblastoma. Proceedings of the National Academy of Sciences of the United States of America 111:291.

Melé M, Ferreira PG, Reverter F, Deluca DS, Monlong J, Sammeth M, Young TR, Goldmann JM, Pervouchine DD, and Sullivan TJ. 2015. The human transcriptome across tissues and individuals. Science 348:660.

Monge C, and León-Velarde F. 1991. Physiological adaptation to high altitude: Oxygen transport in mamals and birds. Physiological Reviews 71:1135-1172.

Nicolas Skuli AJM, Bryan L. Krock, Rickson C. Mesquita, Lijoy K. Mathew, Zachary L. Quinn, Anja Runge, Liping Liu, Meeri N. Kim, Jiaming Liang, Steven Schenkel, Arjun G. Yodh, Brian Keith, M. Celeste Simon. 2012. Endothelial HIF-2 $\alpha$ regulates murine pathological angiogenesis and revascularization processes. Journal of Clinical Investigation 122:14271443.

Nicoli S, Standley C, Walker P, Hurlstone A, Fogarty KE, and Lawson ND. 2010. MicroRNAmediated integration of haemodynamics and Vegf signalling during angiogenesis. Nature 464:1196-1200.

Nivsarkar AE, Gupta SC, Gupta N, Nivsarkar AE, Gupta SC, and Gupta N. 1997. Yak production.

Pruitt KD, Tatusova T, Klimke W, and Maglott DR. 2009. NCBI Reference Sequences: current status, policy and new initiatives. Nucleic Acids Research 37: D32-D36.

Qiu Q, Zhang G, Ma T, Qian W, Wang J, Ye Z, Cao C, Hu Q, Kim J, and Larkin DM. 2012. The yak genome and adaptation to life at high altitude. Nature Genetics 44:946-949.

Remels AH, Gosker HR, Verhees KJ, Langen RC, and Schols AM. 2015. TNF- $\alpha$-induced NF- $\kappa B$ activation stimulates skeletal muscle glycolytic metabolism through activation of HIF-1 $\alpha$. Endocrinology 156:1770-1781.

Robinson MD, Mccarthy DJ, and Smyth GK. 2010. edgeR: a Bioconductor package for differential expression analysis of digital gene expression data. Bioinformatics 26: 139140.

Scaringi R, Piccoli M, Papini N, Cirillo F, Conforti E, Bergante S, Tringali C, Garatti A, Gelfi C, and Venerando B. 2013. NEU3 sialidase is activated under hypoxia and protects skeletal 
436

437

438

439

440

441

442

443

444

445

446

447

448

449

450

451

452

453

454

455

456

457

458

459

460

461

462

463

464 muscle cells from apoptosis through the activation of the epidermal growth factor receptor signaling pathway and the hypoxia-inducible factor (HIF)-1 $\alpha$. The Journal of biological chemistry 288:3153-3162.

Suh SS, Ji YY, Nuovo GJ, Jeon YJ, Kim S, Lee TJ, Kim T, Bakàcs A, Alder H, and Kaur B. 2012. MicroRNAs/TP53 feedback circuitry in glioblastoma multiforme. Proceedings of the National Academy of Sciences of the United States of America 109:5316-5321.

Sun C, Huang C, Li S, Yang C, Xi Y, Wang L, Zhang F, Fu Y, and Li D. 2016. Hsa-miR-326 targetsCCND1 and inhibits non-small cell lung cancer development. Oncotarget 7:83418359.

Sun F, Fu H, Liu Q, Tie Y, Zhu J, Xing R, Sun Z, and Zheng X. 2008. Downregulation of CCND1 and CDK6 by miR-34a induces cell cycle arrest. Febs Letters 582:1564.

Vlachos IS, Zagganas K, Paraskevopoulou MD, Georgakilas G, Karagkouni D, Vergoulis T, Dalamagas T, and Hatzigeorgiou AG. 2015. DIANA-miRPath v3.0: deciphering microRNA function with experimental support. Nucleic Acids Research 43:W460.

Wang H, Long R, Liang JB, Guo X, Ding L, and Shang Z. 2011. Comparison of Nitrogen Metabolism in Yak (Bos grunniens) and Indigenous Cattle (Bos taurus) on the QinghaiTibetan Plateau. Asian Australasian Journal of Animal Sciences 24:766-773.

Wang K, Yang Y, Wang L, Ma T, Shang H, Ding L, Han J, and Qiu Q. 2015. Different gene expressions between cattle and yak provide insights into high-altitude adaptation. Animal Genetics 47:28-35.

Wiener G, Jianlin H, and Ruijun L. 2003. The Yak 2nd edn FAO Regional Office for Asia and the Pacific Food and Agriculture Organization of the United Nations. Bangkok, Thailand.

Xie QH, He XX, Chang Y, Sun SZ, Jiang X, Li PY, and Lin JS. 2011. MiR-192 inhibits nucleotide excision repair by targeting ERCC3 and ERCC4 in HepG2.2.15 cells. Biochemical \& Biophysical Research Communications 410:440-445.

Zhao Q, Shen X, Zhang W, Zhu G, Qi J, and Deng L. 2012. Mice with increased angiogenesis and osteogenesis due to conditional activation of HIF pathway in osteoblasts are protected from ovariectomy induced bone loss. Bone 50:763-770. 


\section{Table $\mathbf{1}$ (on next page)}

Differentially expressed miRNAs in heart and lung tissues between yak and cattle. 
1 Table 1. Differentially expressed miRNAs in heart and lung tissues between yak and cattle

\begin{tabular}{|c|c|c|c|}
\hline \multirow[b]{2}{*}{ Categories } & \multicolumn{3}{|c|}{ Number of differentially expressed miRNAs } \\
\hline & Total & Upregulated in yak & $\begin{array}{l}\text { Upregulated in } \\
\text { cattle }\end{array}$ \\
\hline Heart & 29 & 11 & 18 \\
\hline Lung & 70 & 22 & 48 \\
\hline
\end{tabular}

2 
Table 2 (on next page)

DE miRNAs enriched in P53 signaling pathway. 
1

Table 2. DE miRNAs enriched in P53 signaling pathway

\begin{tabular}{|c|c|c|c|c|}
\hline categories & $\begin{array}{l}\text { miRNA } \\
\text { name }\end{array}$ & heart & lung & Target genes in Tarbase \\
\hline \multirow{6}{*}{$\begin{array}{l}\text { DE in two } \\
\text { tissues }\end{array}$} & $\operatorname{miR}-192-5 p$ & down & down & CDKN2,MDM2, EI24, PERP, ZMAT3 \\
\hline & & & & MDM4, CDK4, ZMAT3, CCNG1, PPM1D, \\
\hline & $\operatorname{miR}-32-3 p$ & down & down & CASP3 \\
\hline & $\operatorname{miR}-200 b-3 p$ & down & up & $\begin{array}{c}\text { MDM4, CCNE1, CDK2, PMAIP1, ZMAT3, } \\
\text { SIAH1, DDB2, RCHY1 }\end{array}$ \\
\hline & $\operatorname{miR}-146 b-5 p$ & up & up & $A T R, C D K N 1 A, T H B S 1, C C N B 3$ \\
\hline & $\operatorname{miR}-122-5 p$ & down & down & $\begin{array}{c}\text { MDM4, ATM, TP53, CCNE1, GTSE1, CD262, } \\
\text { BCL2L4, CCNG1, TP73, CYCS }\end{array}$ \\
\hline \multirow{9}{*}{ DE in heart } & miR-200a-3p & down & no & CDKN1A,CCNE1,SIAH1,THBS1,RRM2B \\
\hline & $\operatorname{miR}-708-5 p$ & down & no & $S E S N 3, C C N B 3$ \\
\hline & $\operatorname{miR}-429$ & down & no & CDK2, PMAIP1, SIAH1 \\
\hline & miR-885-5p & down & no & $C D K 2$ \\
\hline & miR-194-5p & down & no & TSC2 \\
\hline & $\operatorname{miR}-708-3 p$ & down & no & $C A S P 3$ \\
\hline & $\operatorname{miR}-208 a-3 p$ & down & no & CHK1 \\
\hline & $\begin{array}{l}\operatorname{miR}-181 a-2- \\
3 p\end{array}$ & down & no & MDM2,CDK4,GTSE1,BCL2L4,SERPINE1 \\
\hline & miR-218-5p & down & no & $\begin{array}{c}\text { ATR, ATM, TP53, CDKN1A, CDK4, BCL2L4, } \\
\text { ZMAT3, } \\
\text { IGFBP3, SERPINE1, SESN3, CCND3, CD262, } \\
\text { RCHY1 }\end{array}$ \\
\hline \multirow{11}{*}{ DE in lung } & $\operatorname{miR}-424-5 p$ & no & up & $\begin{array}{c}\text { IGFBP3, RRM2B, SESN3, IGFBP3, CCNG1, } \\
\text { SIAH1, MDM2, CHK1, CDKN1A } \\
\text { CDK4, GTSE1, RFWD2, CD262, PERP, ZMAT3, } \\
\text { PPM1D, SCOTIN }\end{array}$ \\
\hline & $\operatorname{miR}-582-5 p$ & no & up & $\begin{array}{c}A T R, C D K 4, C C N D 3, P E R P, S I A H 1, P T E N, \\
C C N G 1, S I A H 1, C Y C S, C C N B 3\end{array}$ \\
\hline & $\operatorname{miR}-34 b-5 p$ & no & up & $\begin{array}{c}C D K N 1 A, C D K 4, C D 262, \text { SIAH1, PPM1D, } \\
\text { THBS1 }\end{array}$ \\
\hline & $\operatorname{miR}-450 b-5 p$ & no & up & MDM2, CDK4, THBS1, SESN3 \\
\hline & $\operatorname{miR}-34 c-5 p$ & no & up & CDK4, STEAP3, SERPINE1 \\
\hline & miR-136-5p & no & up & THBS1 \\
\hline & miR-582-3p & no & up & CDK4, PTEN, CCNB3 \\
\hline & miR-19b-3p & no & up & MDM2,MDM4,ATM,CHK1,TP53,CDKN1A \\
\hline & miR-379-5p & no & down & SERPINE1, CASP3 \\
\hline & miR-382-5p & no & down & PTEN, TSC2, CASP3 \\
\hline & $\operatorname{miR}-181 c-3 p$ & no & down & $M D M 2, E I 24$ \\
\hline
\end{tabular}




$\begin{array}{cccc}\text { miR-299-5p } & \text { no } & \text { down } & \text { CDKN1A, SERPINE1 } \\ \text { miR-449b-5p } & \text { no } & \text { down } & \text { CDK4, SERPINE1, THBS1, STEAP3 } \\ \text { miR-551b-3p } & \text { no } & \text { down } & \text { ZMAT3 } \\ \text { miR-127-3p } & \text { no } & \text { down } & \text { THBS1 } \\ \text { miR-127-5p } & \text { no } & \text { down } & \text { THBS2 } \\ \text { miR-122-3p } & \text { no } & \text { down } & C D 262\end{array}$

2 Note: "down" indicated downregulated in yak; "up" indicated upregulated in yak, "no" 3 indicated not differently expressed in particular tissue.

4 


\section{Figure 1}

The overview of small RNA sequencing.

(A) Mature miRNAs and miRNA precursors identified in this study. (B) Venn charts indicate expression patterns of mature miRNAs among each sample.

A

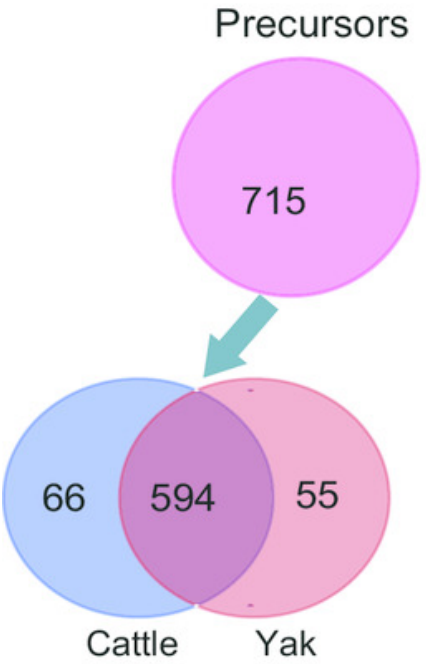

Mature

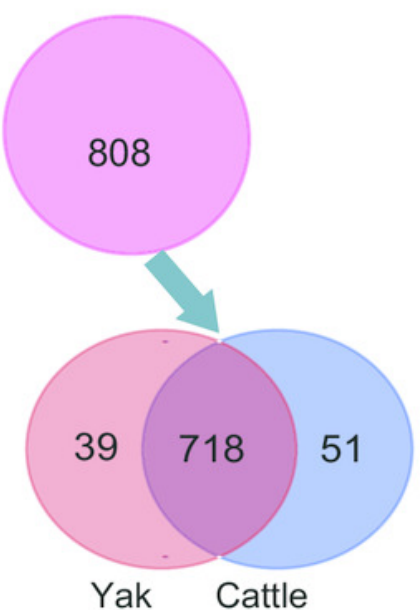

B

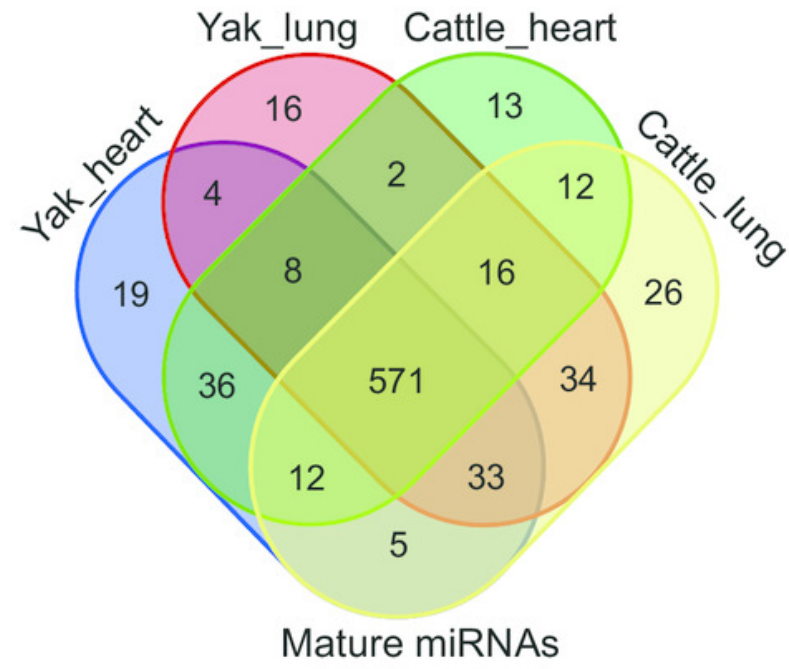




\section{Figure 2}

Figure 2. Highly conserved heart and lung tissues showed varying degrees of divergence.

(A). Hierarchical clustering and heat map matrix of pairwise Spearman's correlations of the counts of 718 co-expressed miRNAs between 11 miRNA libraries. (B). Principal component analysis (PCA) plot of each sample. The fraction of the variance explained is $78 \%$ for eigenvector 1 and 10\% for eigenvector 2. (C). Top 10 unique miRNAs with the highest expression levels in heart and lung tissues of yak and cattle.

A

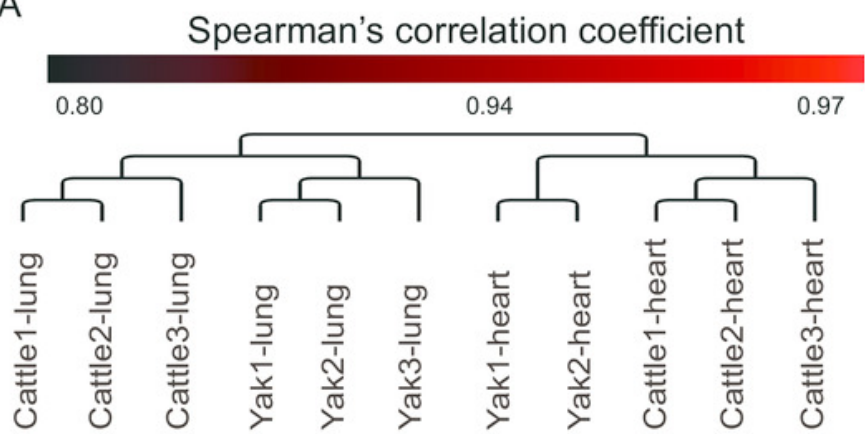

B

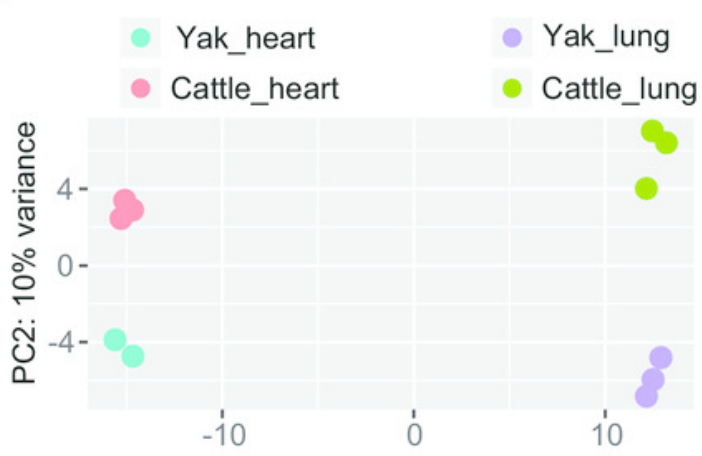

PC1: $78 \%$ variance

C

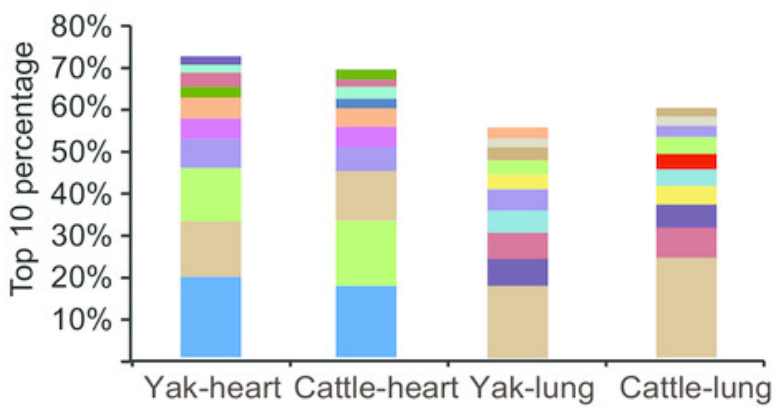

bta-miR-133-3p bta-miR-143-3p bta-miR-22-3p bta-miR-27b-3p ata-miR-499-5p abta-miR-30e-5p bta-miR-486-5p - bta-miR-26a-5p bta-miR-208b-3p bta-miR-30a-5p bta-miR-21-5p bta-miR-26b-5p bta-miR-34c-5p bta-miR-148a-3p bta-miR-126-3p 


\section{Figure 3}

Expression pattern of differentially expressed miRNAs belong to the miRNA family in sequence and miRNA cluster in location.

(A-B) Volcano plot showing differentially expressed miRNAs in heart (A) and lung (B) between yak and cattle. (C) Box plot of Pearson's correlation coefficient for miRNA families, clusters and background. (D) Expression pattern of miR-200 family belonging to miR-200 cluster. (E) Expression pattern of miR-34 family belonging to miR-34 cluster and miR-449 cluster. 

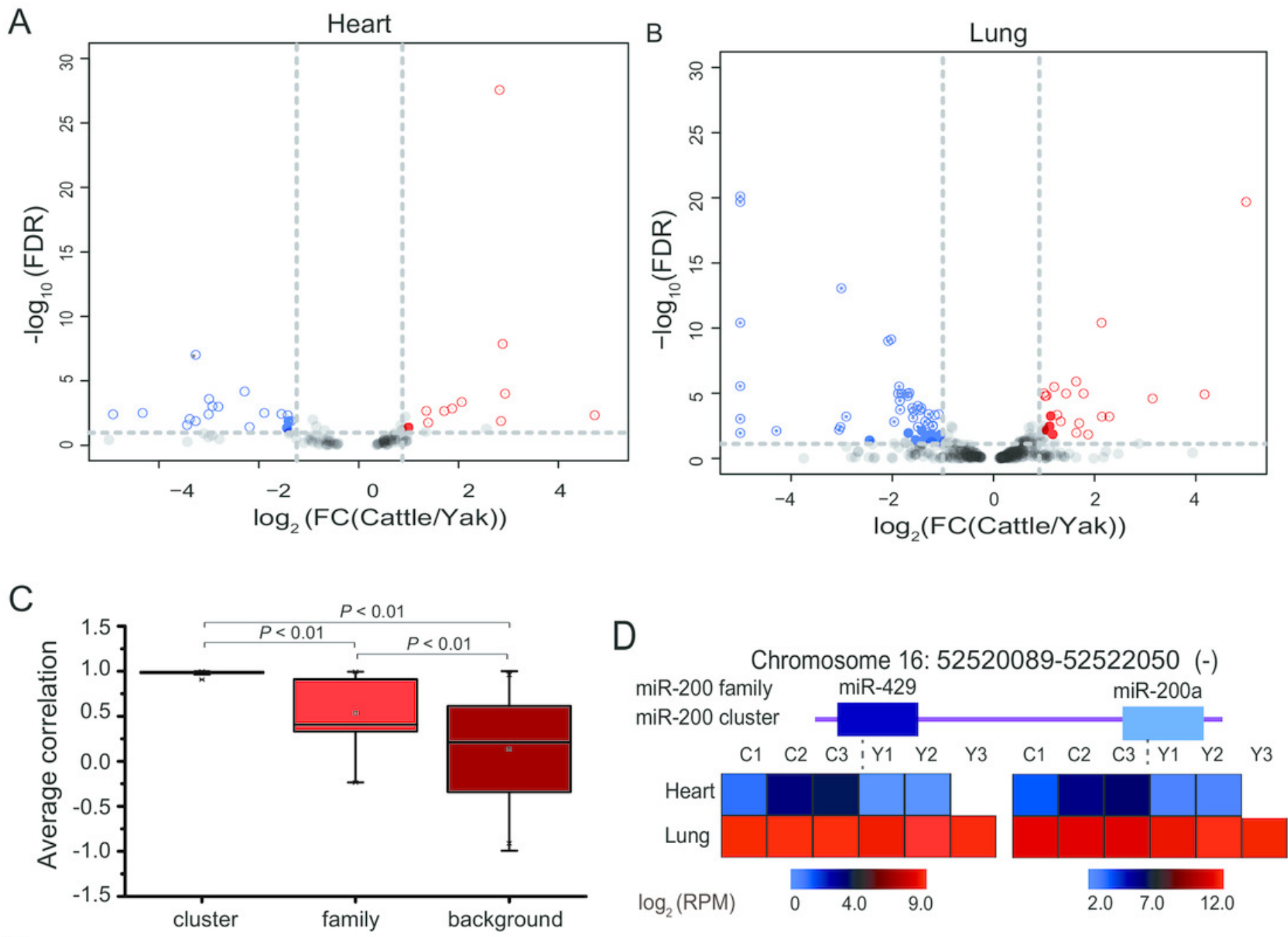

E

Chromosome 15: 22134725-22135502 (+) miR-34 family
miR -34 cluster

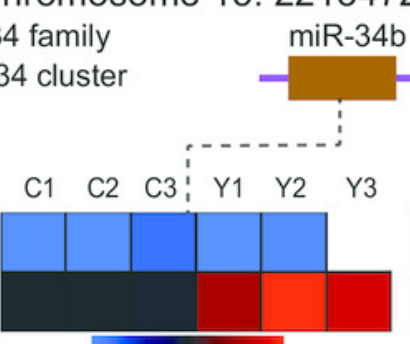

$\log _{2}($ RPM $)$ $\begin{array}{lll}0 & 10.0 & 11.0\end{array}$
Heart

Lung
miR-34c

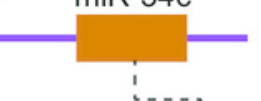

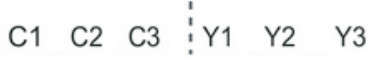

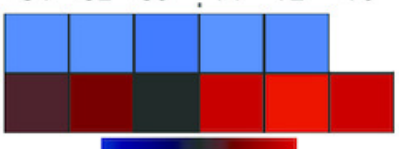

$\begin{array}{lll}0 & 13.0 & 15.0\end{array}$

Chromosome 20: 23967269-23967480 (+)

miR-34 family miR-449 cluster

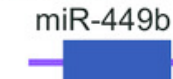

miR-449a

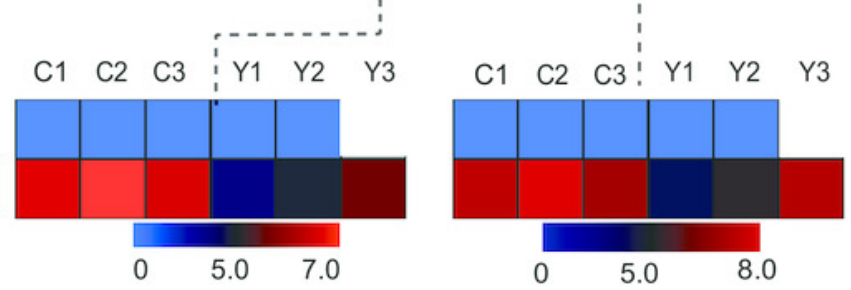




\section{Figure 4}

Figure 4. Functional analysis of DE miRNAs in heart and lung.

(A-B) Gene Ontology (GO) categories and pathways enriched for target genes of DE miRNAs in heart (A) and lung (B). The $p$ value indicating the significance of the enrichment, was corrected by Fisher's exact test. miRNA number was the number of DE miRNAs involved in each $\mathrm{GO}$ term. Gene number was the number of target genes for DE miRNAs involved in each GO term. (C) DE miRNAs involved in p53 feedback circuit. (D) DE miRNAs and corresponding target genes are involved in HIF-1 pathway. miRNAs in red indicated upregulated in yak, miRNAs in black indicated downregulated in yak. 
A

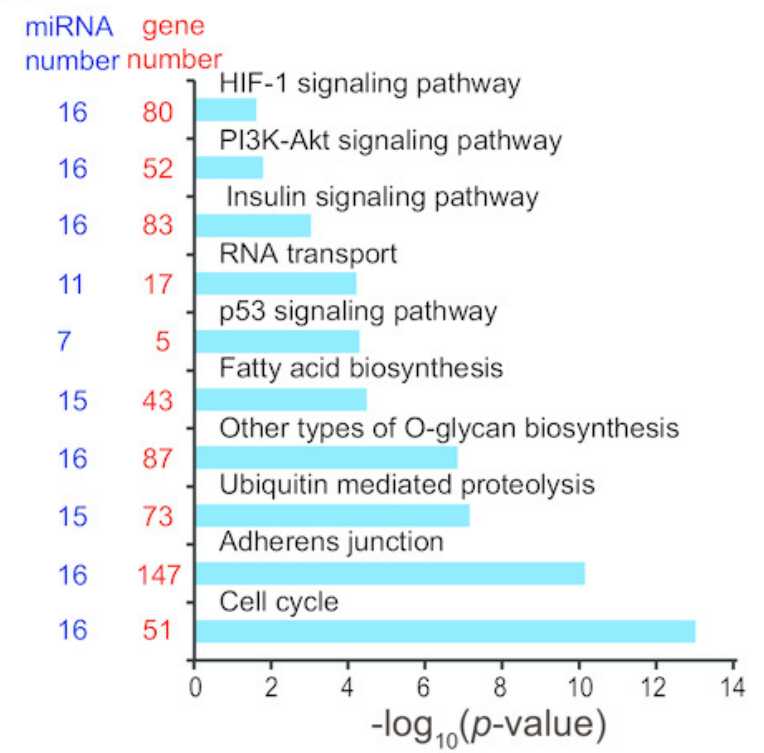

B

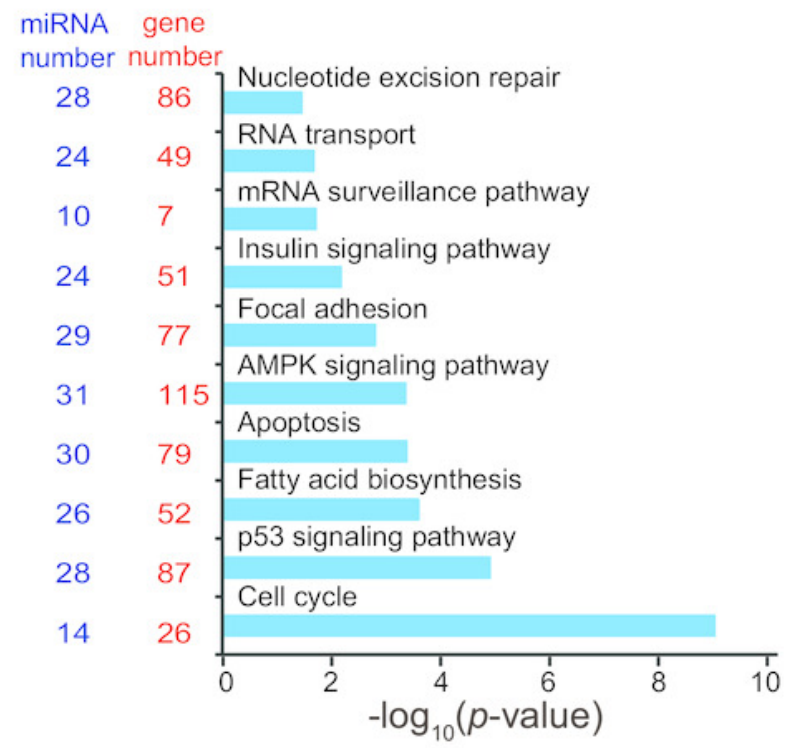

C
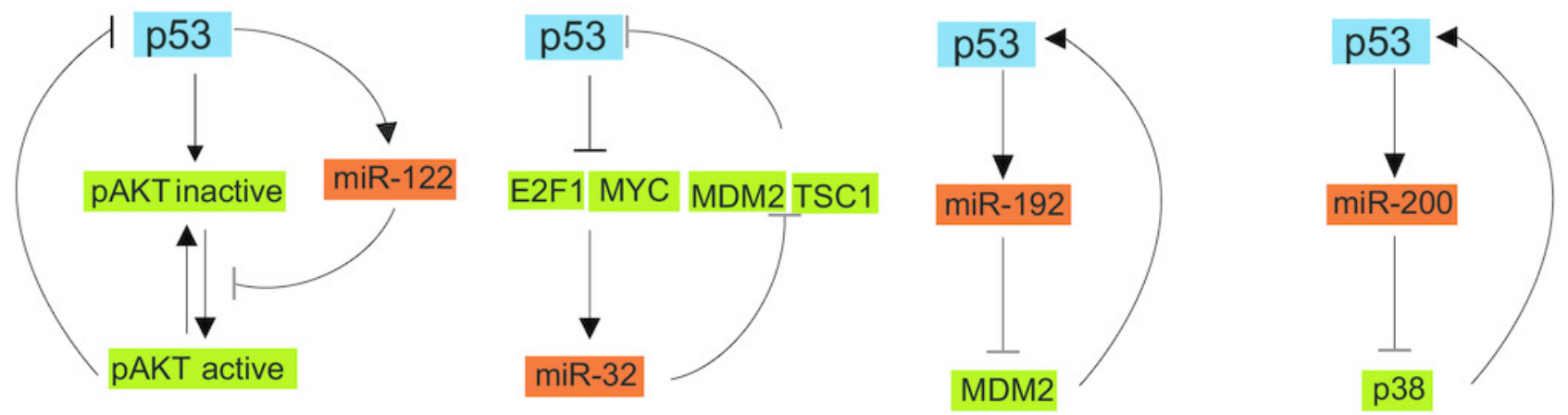

D

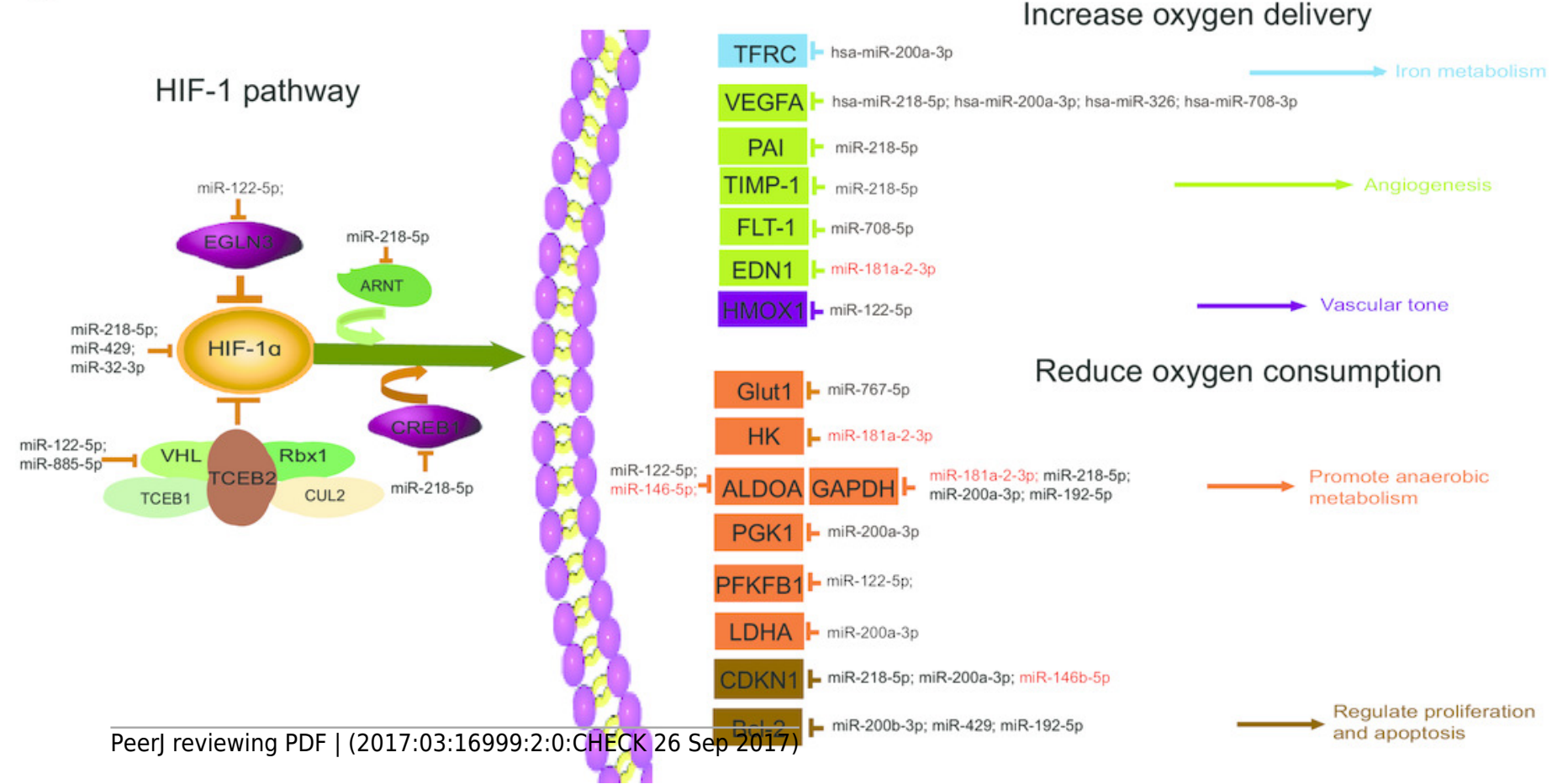

